\title{
Almost All Irritable Bowel Syndromes Are Post-Infectious and Respond to Probiotics: Controversial Issues
}

\author{
Giovanni Barbara Vincenzo Stanghellini Cesare Cremon Roberto De Giorgio \\ Roberto Corinaldesi \\ Department of Internal Medicine and Gastroenterology, University of Bologna, Bologna, Italy
}

\section{Key Words}

Acute infectious gastroenteritis · Irritable bowel syndrome $\cdot$ Post-infectious irritable bowel syndrome • Intestinal microflora, role • Irritable bowel syndrome, clinical management

\begin{abstract}
Acute infectious gastroenteritis is the strongest known risk factor for the development of irritable bowel syndrome (IBS), one of the most common functional gastrointestinal disorders. However, knowledge about the incidence and prevalence of post-infectious IBS (PI-IBS) in the general population is still limited. Some of the published epidemiological studies on PI-IBS lack an appropriate control population, and were limited by a short follow-up symptom assessment post-infection. A number of risk factors have been associated with the development of PI-IBS, including the virulence of the pathogen, younger age, female sex, the long duration of the initial illness and the presence of psychological disturbances. However, much work has to be done to establish whether multifactorial mechanisms actually concur to the pathophysiology of PI-IBS. The discovery that an infective episode may trigger the development of IBS has not substantially changed the clinical management of this subset of patients compared to the classical (non-infective) form of
\end{abstract}

IBS. Probiotics have been claimed to be of some benefit in IBS, but the majority of studies have been performed in nonspecific IBS rather than in PI-IBS and a number of issues still remain to be elucidated.

Copyright $\odot 2007$ S. Karger AG, Basel

\section{Epidemiology}

An acute episode of infectious gastroenteritis may lead, in a small but significant subgroup of patients, to long-term digestive symptoms. In the majority of cases these patients fulfill the criteria for irritable bowel syndrome (IBS). An acute gastrointestinal infection is now a recognized etiological factor for symptom development in a subset of IBS patients, who are defined as being affected by post-infectious IBS (PI-IBS). The epidemiology of PI-IBS remains poorly determined. In particular, little is known about its incidence and prevalence in the general population (and the contribution of infection to IBS). In 1962, Chaudhary and Truelove [1] reported that a proven or highly presumptive episode of acute gastroenteritis could be detected in roughly $25 \%$ of 130 IBS patients referred to their center. More recent data suggest that the prevalence of the infective form of IBS is lower among IBS patients, ranging between 6 and 17\% [2]. This

\section{KARGER \\ Fax +4161306 1234 \\ E-Mail karger@karger.ch}

www.karger.com
(C) 2007 S. Karger AG, Basel

$0257-2753 / 07 / 0253-0245 \$ 23.50 / 0$

Accessible online at:

www.karger.com/ddi
Giovanni Barbara, MD

Department of Internal Medicine and Gastroenterology

St. Orsola-Malpighi Hospital, Building No. 5

Via Massarenti, 9, IT-40138 Bologna (Italy)

Tel. +39 051636 4103, Fax +39 051345 864, E-Mail giovanni.barbara@unibo.it 
wide range of prevalence could be explained by a number of factors, including the different clinical settings in which the studies were carried out (i.e. general population vs. referral centers), the different type of infectious agents involved and the criteria used to diagnose IBS. Furthermore, these data must be considered with caution since they are inevitably retrospective in nature. Indeed, the assessment of a cause-effect relationship between the infection and IBS could be biased by the patient's or physician's need to explain symptoms or by poor recall by the patient of the previous infective episode.

The geographical distribution of PI-IBS is remarkably uneven. PI-IBS has been mainly reported in Europe, particularly in the UK (to date, roughly $70 \%$ of the studies have come from there). Only recently, two studies, one from China [3] and one from Korea [4], have reported that PI-IBS also occurs in Eastern countries with a prevalence similar to that found in the West [5]. On the other hand, older data suggest that PI-IBS is almost absent in developing countries, in spite of a high prevalence of gastrointestinal infections in these populations $[6,7]$. One study from India reported no association between amoebic infection and IBS [8]. To explain this apparent paradox, Gwee [9] has recently proposed a hygiene hypothesis model. According to this hypothesis, it was speculated that in underdeveloped countries a high exposure to a variety of microorganisms early in life could result in a strong stimulation of the intestinal immune system and the development of broad immune tolerance that enable the intestinal epithelium to respond more efficiently to antigenic challenge, as during an episode of gastroenteritis. The consequence would be that post-infective symptoms resolve more quickly. Conversely, the intestinal immune system remains relatively naive in the more hygienic environment of industrialized countries. Gastrointestinal infections in later life would result in a greater inflammatory disturbance, leading to the persistence of IBS symptoms. Further studies are now needed to confirm this interesting hypothesis.

\section{Etiology, Pathophysiology and Risk Factors}

Acute infectious gastroenteritis is a common event in Western countries, occurring on average 1.4 times per year per person. Although the vast majority of subjects recover completely following eviction of the infectious agent, a small but significant proportion of subjects go on to develop long-lasting digestive symptoms [10]. The occurrence of PI-IBS has been confirmed by several pro- spective studies indicating that long-term IBS symptoms develop in $7-32 \%$ of subjects after acute enteritis [11]. One major criticism to these studies is related to the fact that some of them lack a control population, which may lead to an overestimation of the problem. In addition, some studies were carried out with a poor experimental design and limited by a short post-infection follow-up. The fact that only a proportion of patients developed IBS symptoms post-infection suggests the presence of predisposing factors. A number of them have been identified, including virulence of the pathogen, younger age, female sex, long duration of initial illness, and psychological issues (including hypochondriasis and adverse life events) [12]. However, much work is needed to elucidate the exact role of these factors.

The fact that IBS may have an infective origin has created renewed interest and enthusiasm in IBS research. One may even postulate some analogies with the infective (i.e. Helicobacter pylori) origin of peptic ulcer disease. However, the scenario of PI-IBS is remarkably different. The variety of infective agents involved in the development of IBS symptoms clearly indicates that the etiology is rather variable and therefore the disease is not the consequence of any specific infection. The infectious agents more frequently found to be associated with PIIBS are Campylobacter, Shigella and Salmonella [10]. Other bacterial agents including viruses and parasites could possibly be involved in the development of PI-IBS; however, supporting data are still lacking. The acute infective episode can be considered only a trigger factor involved in the stimulation of a local (i.e. intestinal) inflammatory response. Patients that go on to develop IBS symptoms post-infection are probably unable to downregulate efficiently this initial inflammatory response. Genetic and psychological factors [11] as well as the use of antibiotics [13] during the acute infective episode have been claimed to be involved in the persistence of the intestinal lowgrade inflammation.

In support of the hypothesis that low-grade intestinal inflammation participated in symptom generation is evidence that other conditions characterized by local lowgrade immune activation such as ulcerative colitis in remission [14] or treated celiac disease [15] are also associated with the development of IBS-like symptoms. Although these studies support the role of inflammatory mechanisms in symptom generation in PI-IBS patients, a clear demonstration of a cause-effect relationship is still awaited. 


\section{Role of Intestinal Microflora}

Some studies have provided evidence that intestinal microflora may be altered in patients with IBS. These changes may contribute to the low-grade intestinal inflammatory response described in PI-IBS. However, the role of these changes in the pathophysiology of PI-IBS is still controversial. This is related to our limited knowledge of the intestinal microflora even in healthy human subjects. This has to be ascribed to human microflora complexity, variability and instability and to substantial methodological flaws that hamper an in-depth characterization of indigenous intestinal flora. A more detailed definition of intestinal bacterial populations could be obtained by new molecular techniques such as polymerase chain reaction, quantitative dot-blot, fluorescence in situ hybridization, and DNA microarray [16]. A detailed characterization of intestinal microflora using these newer techniques will most likely require several years of work.

Other potential mechanisms possibly involved in the pathophysiology of PI-IBS include changes in serotonin metabolism [10] and the occurrence of an increased postinfection bile acid malabsorption [17], but further confirmatory studies are required.

\section{Clinical Management}

The discovery that an infective episode may trigger the development of IBS has not substantially changed the clinical management of this subset of patients compared to the classical (non-infective) form of IBS. Indeed, there are no clear preventive measures in PI-IBS. An initial attempt to reduce intestinal inflammation with steroids in PI-IBS has failed to demonstrate any symptomatic improvement [18]. Nonetheless, these apparently disap- pointing results should be viewed in light of the known side effects of steroids, including those affecting the gastrointestinal tract. Larger trials and eventually testing other anti-inflammatory compounds may provide some better beneficial effect in PI-IBS.

Non-absorbable antibiotics (e.g. rifaximin) have been recently shown to be beneficial in the treatment of nonspecific IBS [19]. Nonetheless, to date there are no available studies assessing a similar approach in PI-IBS. Probiotics have been claimed to be of some benefit in IBS, but the majority of studies have been carried out in non-specific IBS rather than in PI-IBS, conflicting results have also been published [16] and a number of issues still remain to be elucidated. These include the identification of subsets of patients who respond to this type of therapy, better definition of outcome measures, identification of the most effective type of probiotic formulation (e.g. type of strain, multiple vs. single strain, live vs. non-live, spores, yoghurts) and the most effective dose of probiotics. Much work is obviously needed in this interesting field of investigation.

The prognosis of PI-IBS has been suggested to be slightly better to that of non-specific IBS [1]. A 6-year follow-up study in 192 patients showed that symptoms disappeared in $43 \%$ of PI-IBS as compared to $31 \%$ in nonspecific IBS, and, although this difference did not reach statistical significance, a type B error cannot be excluded [20].

In conclusion, acute infectious gastroenteritis is an accepted etiological factor in IBS. However, a number of controversial issues regarding its epidemiology and pathophysiology remain to be clarified. For the time being, the clinical management of this subgroup of patients is not different from that of non-specific IBS. Although probiotics hold a great therapeutic promise for patients with PI-IBS, their exact role in the management of these patients needs to be elucidated.

\section{References}

1 Chaudhary NA, Truelove SC: The irritable colon syndrome. A study of the clinical features, predisposing causes, and prognosis in 130 cases. Q J Med 1962;31:307-322.

-2 Longstreth GF, Hawkey CJ, Mayer EA, Jones RH, Naesdal J, Wilson IK, Peacock RA, Wiklund IK: Characteristics of patients with irritable bowel syndrome recruited from three sources: implications for clinical trials. Aliment Pharmacol Ther 2001;15:959-964.

Almost All IBS Are Post-Infectious and Respond to Probiotics
-3 Wang LH, Fang XC, Pan GZ: Bacillary dysentery as a causative factor of irritable bowel syndrome and its pathogenesis. Gut 2004;53: 1096-1101.

-4 Ji S, Park H, Lee D, Song YK, Choi JP, Lee SI: Post-infectious irritable bowel syndrome in patients with Shigella infection. J Gastroenterol Hepatol 2005;20:381-386.

5 Collins SM, Barbara G: East meets West: infection, nerves, and mast cells in the irritable bowel syndrome. Gut 2004;53:1068-1069.
6 Anand AC, Reddy PS, Saiprasad GS, Kher SK: Does non-dysenteric intestinal amoebiasis exist? Lancet 1997;349:89-92.

7 Nanda R, Baveja U, Anand BS: Entamoeba histolytica cyst passers: clinical features and outcome in untreated subjects. Lancet 1984; 2:301-303

-8 Sinha P, Ghoshal UC, Choudhuri G, Naik S, Ayyagari A, Naik SR: Does Entamoeba histolytica cause irritable bowel syndrome? Indian J Gastroenterol 1997;16:130-133. 
9 Gwee KA: Irritable bowel syndrome in developing countries - a disorder of civilization or colonization? Neurogastroenterol Motil 2005;17:317-324.

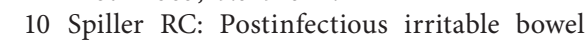
syndrome. Gastroenterology 2003; 124: 1662-1671.

11 Barbara G, De Giorgio R, Stanghellini V, Cremon C, Salvioli B, Corinaldesi R: New pathophysiological mechanisms in irritable bowel syndrome. Aliment Pharmacol Ther 2004;20(suppl 2):1-9.

$\checkmark 12$ Neal KR, Hebden J, Spiller R: Prevalence of gastrointestinal symptoms six months after bacterial gastroenteritis and risk factors for development of the irritable bowel syndrome: postal survey of patients. BMJ 1997; 314:779-782.
13 Barbara G, Stanghellini V, Berti-Ceroni C, De Giorgio R, Salvioli B, Corradi F, Cremon C, Corinaldesi R: Role of antibiotic therapy on long-term germ excretion in faeces and digestive symptoms after Salmonella infection. Aliment Pharmacol Ther 2000;14: 1127-1131.

14 Isgar B, Harman M, Kaye MD, Whorwell PJ: Symptoms of irritable bowel syndrome in ulcerative colitis in remission. Gut 1983;24: 190-192.

15 Fine KD, Meyer RL, Lee EL: The prevalence and causes of chronic diarrhea in patients with celiac sprue treated with a gluten-free diet. Gastroenterology 1997;112:1830-1838.

16 Barbara G, Stanghellini V, Brandi G, Cremon C, Di Nardo G, De Giorgio R, Corinaldesi R: Interactions between commensal bacteria and gut sensorimotor function in health and disease. Am J Gastroenterol 2005; 100:2560-2568.
17 Niaz SK, Sandrasegaran K, Renny FH, Jones BJ: Postinfective diarrhoea and bile acid malabsorption. J R Coll Physicians Lond 1997;31:53-56.

18 Dunlop SP, Jenkins D, Neal KR, Naesdal J, Borgaonker M, Collins SM, Spiller RC: Randomized, double-blind, placebo-controlled trial of prednisolone in post-infectious irritable bowel syndrome. Aliment Pharmacol Ther 2003;18:77-84.

19 Pimentel M, Park S, Mirocha J, Kane SV, Kong Y: The effect of a nonabsorbed oral antibiotic (rifaximin) on the symptoms of the irritable bowel syndrome: a randomized trial. Ann Intern Med 2006;145:557-563.

20 Neal KR, Barker L, Spiller RC: Prognosis in post-infective irritable bowel syndrome: a six-year follow-up study. Gut 2002;51:410413. 